\title{
Finite temperature properties of a modified Polyakov-Nambu-Jona-Lasinio model
}

\author{
Abhijit Bhattacharyya* \\ Department of Physics, University of Calcutta, 92, A.P.C Road, Kolkata-700009, India \\ Paramita Deb $\oplus^{\dagger}$ \\ Indian Institute of Technology Bombay, Mumbai-400076, India \\ Sanjay K. Ghosh $\odot,{ }^{\ddagger}$ Soumitra Maity, ${ }^{\S}$ Sibaji Raha,, and Rajarshi Ray® \\ Center for Astroparticle Physics \& Space Science, Block-EN, Sector-V, Salt Lake, Kolkata-700091, India \\ and Department of Physics, Bose Institute, 93/1, A. P. C Road, Kolkata-700009, India \\ Kinkar Saha $\oplus^{* *}$ \\ Department of Physics, University of Calcutta, 92, A.P.C Road, Kolkata-700009, India \\ Sudipa Upadhaya ${ }^{\dagger \dagger}$ \\ Variable Energy Cyclotron Centre, I/AF, Bidhannagar, Kolkata-700064, India
}

(Received 19 December 2019; accepted 10 September 2020; published 13 October 2020)

\begin{abstract}
Thermodynamic properties of strongly interacting matter are investigated using the Polyakov loop enhanced Nambu-Jona-Lasinio model along with some modifications to include the hadrons. Various observables are shown to have a close agreement with the numerical data of QCD on lattice. The advantage of the present scheme over a similar study using a switching function is that here no extra parameters are to be fitted. As a result the present scheme can be easily extended for finite chemical potentials.
\end{abstract}

DOI: 10.1103/PhysRevD.102.074006

\section{INTRODUCTION}

The program of various ongoing and upcoming relativistic heavy-ion collision experiments is to explore the properties of strongly interacting matter at finite temperatures and densities. There have been major theoretical advances in the finite temperature properties using simulations of quantum chromodynamics (QCD) on space-time lattices. Currently the temperature $T_{c}$ where a rapid crossover from hadronic to partonic matter takes place is found to be close to the pion mass. The reported values are $155 \mathrm{MeV}$ [1,2] and $150 \mathrm{MeV}$ [3] from HotQCD and

\footnotetext{
*abphy@caluniv.ac.in

paramita.deb83@gmail.com

*sanjay@jcbose.ac.in

\$soumitra.maity1984@gmail.com

"sibaji@jcbose.ac.in

rajarshi@jcbose.ac.in

saha.k.09@gmail.com

sudipa.09@gmail.com
}

Published by the American Physical Society under the terms of the Creative Commons Attribution 4.0 International license. Further distribution of this work must maintain attribution to the author(s) and the published article's title, journal citation, and DOI. Funded by SCOAP ${ }^{3}$.
Wuppertal-Budapest (WuB) collaborations respectively. At the same time various QCD inspired model frameworks have been developed to extract interesting physical insights. The Nambu-Jona-Lasinio or the NJL model [4-9] is one such model which effectively incorporates key features of QCD like chiral symmetry breaking and its restoration. However the effects of the gluon degrees of freedom are not adequately addressed in such models. Extensions like the Polyakov loop enhanced Nambu-Jona-Lasinio (PNJL) model [10-14] encapsulate this missing feature by including a temporal background gluon field. As a result, both chiral and deconfinement aspects are captured within a single framework. Various studies of the basic thermodynamic variables computed in the mean field framework display strong similarity to lattice results (see [15] and references therein).

With more sophisticated techniques and higher computational power, some of the observables from lattice QCD have been extrapolated to the physical continuum limit $[2,3]$. Many of these results are quite different from those obtained earlier on smaller lattices. In view of this, a reparametrization of the PNJL model was done [16] to obtain a quantitative agreement with the lattice QCD data. One important lacuna observed in this study is the mismatch of the results for temperatures close to or below 
$T_{c}$. The reason was identified as the absence of hadronic contribution in the PNJL model. Several other attempts were going on to construct a suitable model to match the lattice data. In HRG + chiral perturbation theory [17], below the transition temperature, the decrease of the absolute value of chiral condensate is well described. Also HRG model is able to reproduce LQCD data on temperature dependence of the Polyakov-loop itself [18]. Similarly, a quark-hadron hybrid model [19] has been constructed by taking quark and hadron contributions simultaneously. The hadron volume fraction function is used to switch from one phase to other and hadron-quark transition temperature is defined in the view of the ratio of quark and hadron contribution. In this direction some of us studied a hybrid model by coupling the HRG model and the PNJL model via a switching function [15].

Here we study an alternative scheme where the hadron contributions are added in a simple way except that we consider their medium dependent masses. The confinement feature through the Polyakov loop always suppresses the contribution of the constituent quarks at low temperatures and densities. The switching function was necessary to rather cut off the contribution of the hadrons at high temperatures and densities. Instead of the switching function, here the rising effective masses of the hadrons will naturally make them unfavorable in the thermodynamics. We focus on the region of vanishing chemical potential to see the proximity with lattice QCD dynamics, before we move toward more complicated scenarios like those of finite chemical potentials in our upcoming projects. This framework will then be very useful and effective as there are no extra parameters to be dealt with and the information of temperatures and chemical potentials can be taken care of in respective distribution functions.

In the next section, we briefly outline the PNJL model. The following section gives a description of how we handle the hadrons. This is followed by our results and conclusions.

\section{PNJL MODEL}

We now discuss the particular form of the PNJL model as discussed in Ref. [16], which will be employed here. The scheme in the PNJL model was to add a Polyakov loop effective potential to the NJL model $[10,12,20]$. The chiral properties are taken care of by the NJL part, while the confinement properties and the gluonic contributions are effectively incorporated through the Polyakov loop potential. Various studies have been carried out using PNJL model with 2 and $2+1$ flavors [12,21-30]. For our study we shall use the $2+1$ flavor model having up to six quark interactions. The thermodynamic potential is given as [16],

$$
\begin{aligned}
\Omega\left(\Phi, \bar{\Phi}, \sigma_{f}, T, \mu\right)= & 2 g_{S} \sum_{f=u, d, s} \sigma_{f}^{2}-\frac{g_{D}}{2} \sigma_{u} \sigma_{d} \sigma_{s}-6 \sum_{f} \int_{0}^{\Lambda} \frac{d^{3} p}{(2 \pi)^{3}} E_{f} \Theta(\Lambda-|\vec{p}|) \\
& -2 T \sum_{f} \int_{0}^{\infty} \frac{d^{3} p}{(2 \pi)^{3}} \ln \left[1+3\left(\Phi+\bar{\Phi} e^{-\left(E_{f}-\mu_{f}\right) / T}\right) e^{-\left(E_{f}-\mu_{f}\right) / T}+e^{-3\left(E_{f}-\mu_{f}\right) / T}\right] \\
& -2 T \sum_{f} \int_{0}^{\infty} \frac{d^{3} p}{(2 \pi)^{3}} \ln \left[1+3\left(\bar{\Phi}+\Phi e^{-\left(E_{f}+\mu_{f}\right) / T}\right) e^{-\left(E_{f}+\mu_{f}\right) / T}+e^{-3\left(E_{f}+\mu_{f}\right) / T}\right] \\
& +\mathcal{U}^{\prime}(\Phi, \bar{\Phi}, T) .
\end{aligned}
$$

The first five terms on the right-hand side (rhs) are the terms of the NJL model suitably modified due to the Polyakov loop. Here $\sigma_{f}=\left\langle\bar{\psi}_{f} \psi_{f}\right\rangle$ correspond to the two light quark $(f=u, d)$ condensates and the strange $(f=s)$ quark condensate respectively. There is a four quark coupling term with coefficient $g_{S}$ and a six quark coupling term breaking the axial U(1) symmetry explicitly with a coefficient $g_{D}$. The corresponding quasiparticle energy is $E_{f}=\sqrt{p^{2}+M_{f}^{2}}$, for a given flavor $f$. The dynamically generated constituent quark masses are given by,

$$
M_{f}=m_{f}-2 g_{S} \sigma_{f}+\frac{g_{D}}{2} \sigma_{f+1} \sigma_{f+2},
$$

where, if $\sigma_{f}=\sigma_{u}$, then $\sigma_{f+1}=\sigma_{d}$ and $\sigma_{f+2}=\sigma_{s}$, and so on in a clockwise manner.

The third term on the rhs of Eq. (1) gives the zero point energy, while the fourth and fifth terms are the finite temperature and chemical potential contributions of the constituent quarks and antiquarks respectively. The latter two terms arise from the fermion determinant in the NJL model duly modified by the fields corresponding to the traces of Polyakov loop and its conjugate given by $\Phi=\frac{T r_{c} L}{N_{c}}$ and $\bar{\Phi}=\frac{T r_{c} L^{\dagger}}{N_{c}}$ respectively. Here $L(\vec{x})=\mathcal{P} \exp \left[i \int_{0}^{1 / T} d \tau A_{4}(\vec{x}, \tau)\right]$ is the Polyakov loop, and $A_{4}$ is the temporal component of background gluon field.

The effective potential for the $\Phi$ and $\bar{\Phi}$ fields are given by $\mathcal{U}^{\prime}$, appearing as the last term in Eq. (1). Various forms of the potential exist in the literature (see, e.g., [23,31-34]). We shall use the form prescribed in [16] which reads as,

$$
\frac{\mathcal{U}^{\prime}(\Phi, \bar{\Phi}, T)}{T^{4}}=\frac{\mathcal{U}(\Phi, \bar{\Phi}, T)}{T^{4}}-\kappa \ln [J(\Phi, \bar{\Phi})] .
$$

Here $\mathcal{U}(\Phi, \bar{\Phi}, T)$ chosen as a Landau-Ginzburg type potential commensurate with the global Z(3) symmetry of the Polyakov loop is given as [12], 
$\frac{\mathcal{U}(\Phi, \bar{\Phi}, T)}{T^{4}}=-\frac{b_{2}(T)}{2} \bar{\Phi} \Phi-\frac{b_{3}}{6}\left(\Phi^{3}+\bar{\Phi}^{3}\right)+\frac{b_{4}}{4}(\bar{\Phi} \Phi)^{2}$.

The coefficient $b_{2}(T)$ is chosen to have a temperature dependence of the form [16],

$$
b_{2}(T)=a_{0}+a_{1} \exp \left(-a_{2} \frac{T}{T_{0}}\right) \frac{T_{0}}{T},
$$

and $b_{3}$ and $b_{4}$ are chosen to be constants. The term $J[\Phi, \bar{\Phi}]=\left(1-6 \bar{\Phi} \Phi+4\left(\bar{\Phi}^{3}+\Phi^{3}\right)-3(\bar{\Phi} \Phi)^{2}\right) \quad$ is the Jacobian of transformation from the Polyakov loop to its traces. $\kappa$ is a dimensionless parameter which is determined phenomenologically.

The different parameter values in the NJL terms are given in Table I. And the parameter values used in the Polyakov loop potential are given in Table II.

Previously some of us [16] discussed that this model gives a crossover temperature of $T_{c} \sim 160 \mathrm{MeV}$ as well as quantitative agreement of temperature variations of pressure and various other observables commensurate with the observations in lattice QCD in the continuum limit [16]. However the quantitative agreement though close, was not exact in different ranges of temperatures. Significant discrepancies appeared in the low temperature region where the hadronic degrees of freedom dominate. A possible step toward removal of this lacuna was proposed by us [15] by coupling the PNJL model with the hadron resonance gas model via a switching function. This scheme was successful in getting a much better agreement between the results from PNJL model and the lattice QCD data. Here a key role is played by the switching function that switches the hadronic or the partonic degrees of freedom. However this approach requires us to immaculately choose a form and parametrization of the switching function itself. Here we discuss an alternative scheme where the PNJL model is modified such that the hadronic contributions would appear more naturally in the relevant region of the phase space and shut off in other regions, without having to use a switching by hand. In the next section we shall describe this scheme.

TABLE I. Parameters in the NJL model.

\begin{tabular}{lcccc}
\hline \hline$m_{u}(\mathrm{MeV})$ & $m_{s}(\mathrm{MeV})$ & $\Lambda(\mathrm{MeV})$ & $g_{s} \Lambda^{2}$ & $g_{D} \Lambda^{5}$ \\
\hline 5.5 & 134.758 & 631.357 & 3.664 & 74.636 \\
\hline \hline
\end{tabular}

TABLE II. Parameters for the Polyakov loop potential.

\begin{tabular}{lcccccc}
\hline \hline$T_{0}(\mathrm{MeV})$ & $a_{0}$ & $a_{1}$ & $a_{2}$ & $b_{3}$ & $b_{4}$ & $\kappa$ \\
\hline 175 & 6.75 & -9.0 & 0.25 & 0.805 & 7.555 & 0.1 \\
\hline \hline
\end{tabular}

\section{HADRONIC SECTOR}

Our aim is to include all the correct degrees of freedom allowed in strong interactions in our model framework. As discussed in [15] (and references therein), the prominent degrees of freedom would depend on the thermodynamic conditions. This gave a scope for introducing the phenomenologically determined switching function in [15], and couple the PNJL model to the HRG model. Here we ask if a more natural mechanism exists to include the hadronic contributions. Here we have considered all hadrons with masses up to $1 \mathrm{GeV}$. We have explicitly checked that hadrons having masses higher than $1 \mathrm{GeV}$ do not contribute to the thermodynamic quantities significantly.

As is well known, the thermodynamic potential given by Eq. (1) is obtained in the mean field approximation for the quark propagators. A consistent method to extract the thermodynamic potential beyond mean field for a quark meson plasma in the framework of the NJL model was outlined in [35-37]. The mesonic contributions appear in the next to leading order contributions in a $1 / N_{c}$ expansion in the form of ring diagrams. For a meson $M$, the contribution to the thermodynamic potential is given by,

$$
\begin{aligned}
\delta \Omega_{M}= & g_{M} \int \frac{d^{3} p}{(2 \pi)^{3}} \int d \omega\left[\frac{\omega}{2}+T \ln \left(1-e^{-\frac{\omega}{T}}\right)\right] \\
& \times \frac{1}{\pi} \frac{d \delta_{M}(\omega, \vec{p}, T)}{d \omega} .
\end{aligned}
$$

Here, $g_{M}$ is the internal degrees of freedom of the meson and $\delta_{M}(\omega, \vec{p}, T)$ is the scattering phase shift of a quark and antiquark in the $M$ channel.

Extensions of this work in the PNJL model have been done in [38-42], wherein the authors have studied various effects of this additional contribution to the mean-field thermodynamic potential. On the other hand here we set out to make a detailed study of the various thermodynamic observables and contrast them to the results reported in the continuum limit in the lattice QCD framework. Here we do not try to be rigorous with the beyond mean-field calculations but carry out a simple heuristic approach. We simply add the hadronic contribution to the mean field PNJL model. The masses of such hadronic excitations may be computed from the pole condition in the respective polarizations, and would therefore depend implicitly on the mean fields and explicitly on the thermodynamic parameters. This new framework therefore entwines the two phases of QCD matter in a somewhat interactive and medium-dependent way. This approach is similar to the near-pole approximation $\left(\omega^{2}=E_{M}^{2}=\vec{p}^{2}+m_{M}^{2}\right)$ of the above thermodynamic potential [35]. In practice this approach is similar to our earlier approach [15] of adding the hadronic contribution to the PNJL model, but without a switching function. Here the effect of switching off/on of the hadronic contributions will rather be taken care of by 
the relative strength of the temperature dependent hadronic masses to the quark masses. This approach would also help to judge the amount of contributions from hadronic sector. In addition, this would also provide us an idea whether adding strange mesons only to the picture, can address the discrepancies in the strange sector.

The temperature dependent mesonic masses are obtained from the pole condition

$$
1-2 G_{M} \Pi_{M}\left(\omega=m_{M}, \vec{k}=0\right)=0 .
$$

Here $G_{M}$ is the effective vertex factor for the given flavor combination and $\Pi_{M}\left(k^{2}\right)$ is the one-loop polarization function for corresponding mesonic channel given by the random phase approximation [43] as,

$\Pi_{M}\left(k^{2}\right)=\int \frac{d^{4} p}{(2 \pi)^{4}} \operatorname{Tr}\left[\Gamma_{M} S\left(p+\frac{k}{2}\right) \Gamma_{M} S\left(p-\frac{k}{2}\right)\right]$,

where $S(p)$ is the quark propagator. In this work we shall only consider the lowest lying nonet mesons. The details of the calculations may be found in our earlier work $[44,45]$. The final computations however consider the reparametrized PNJL model as discussed in the previous section. The mesonic contribution to the thermodynamic potential is given as [31],

$$
\delta \Omega_{M}=-\nu_{M} T \int \frac{d^{3} p}{(2 \pi)^{3}} \ln \left(1-e^{-\frac{E_{p}}{T}}\right)
$$

where $\nu_{M}$ is the statistical weight factor of the corresponding mesonic species and $E_{p}=\sqrt{\vec{p}^{2}+m_{\text {pole }}^{2}(T)}$, where $m_{\text {pole }}$ is the mesonic mass obtained by solving Eq. (7).

In the baryonic sector, the lower lying window is occupied by the nucleons, protons and neutrons. They, having a bare mass $\sim 940 \mathrm{MeV}$, contribute insignificantly to the thermodynamics. Also, chiral perturbation theory results like in [46] indicate that the nucleon masses increase with temperature apart from a very small decrease in the intermediate regimes of temperature. In this study we therefore consider only constant mass for nucleons. The role of other baryon species is left out in this exercise. The baryonic contribution to the thermodynamic potential is given by [47],

$$
\delta \Omega_{B}=\nu_{B} T \int \frac{d^{3} p}{(2 \pi)^{3}} \ln \left(1+e^{-\frac{E_{p}}{T}}\right)
$$

where $\nu_{B}$ is the statistical weight factor of the corresponding baryonic species. The final thermodynamic potential is the sum of the parts obtained from Eq. (1), Eq. (9) and Eq. (10). We shall refer to this as the modified Polyakov loop enhanced Nambu-Jona-Lasinio (MPNJL) model.

\section{RESULTS}

The thermodynamic potential (1) is minimized with respect to $\sigma, \Phi$ and $\bar{\Phi}$ to obtain the mean fields. These are then inserted into Eqs. (7) and (8) to obtain the meson masses as functions of temperature. In Fig. 1 we have plotted the variations of masses for $\pi, \sigma, K, \eta$, and $\eta^{\prime}$ with temperature. The results are similar to some of the earlier works $[44,48]$. The most significant change is the mass of $\pi$ which rises from about $140 \mathrm{MeV}$ near the crossover temperature to $550 \mathrm{MeV}$ as the temperature nears $400 \mathrm{MeV}$. The $\sigma$ mass has the expected behavior of first a strong decrease to reach the $\pi$ mass and then increase along with the $\pi$ mass. In this temperature range, the $\eta^{\prime}$ mass decreases by almost $300 \mathrm{MeV}$. The masses of $K$ and $\eta$ vary by relatively small amounts. The various combinations of constituent quark masses are also plotted for comparison. Obviously the signature of the chiral symmetry restoration in the meson sector at high temperatures is evident as the constituent masses go down. In this work the constituent quark mass approaches the current mass at about $365 \mathrm{MeV}$ and the mass of $\pi$ approaches the mass of $\eta$ at a temperature of about $435 \mathrm{MeV}$. This result is somewhat different from that obtained in [49], where the authors have investigated the effects of different cutoffs on the meson masses and hence analyzed the effective restorations of chiral and axial symmetries using NJL model. There, both the temperatures are found to be $333 \mathrm{MeV}$. However, it is to be noted that we have used a different model, namely reparametrized version of the PNJL model [16], with the model parameters, even in the NJL sector, being different.

The mean fields are then put back in Eq. (1), and the pole masses in Eq. (9) and Eq. (10) to obtain the pressure.

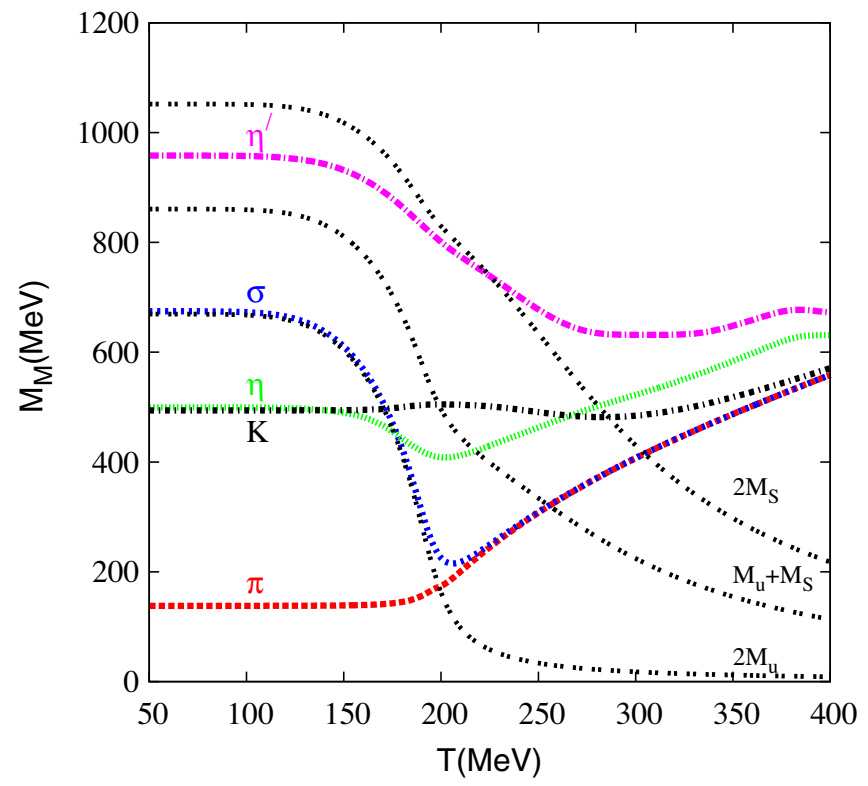

FIG. 1. Pseudoscalar and scalar meson masses plotted as a function of temperature. 


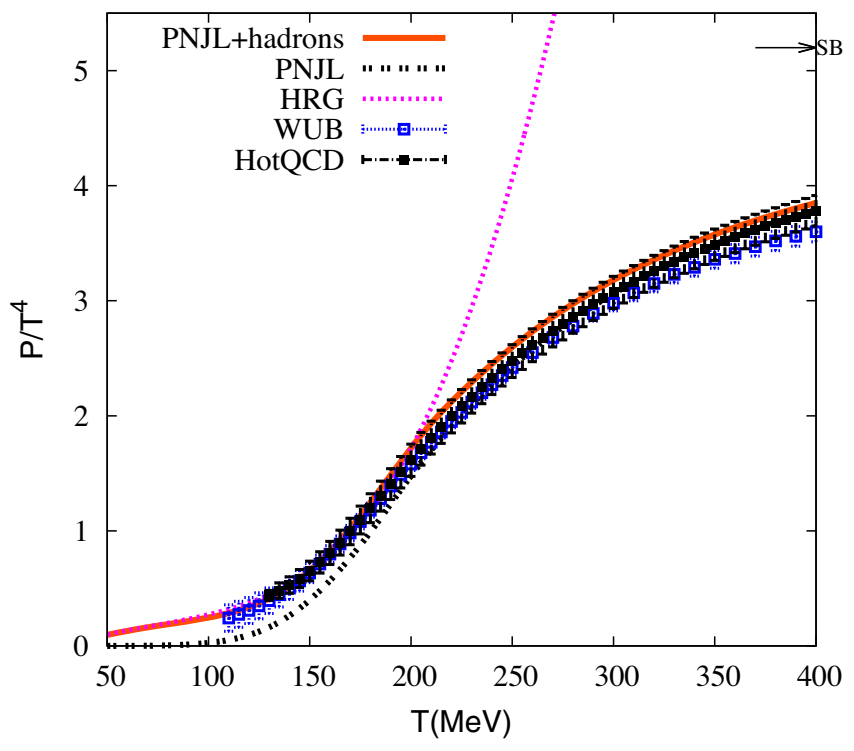

(a)

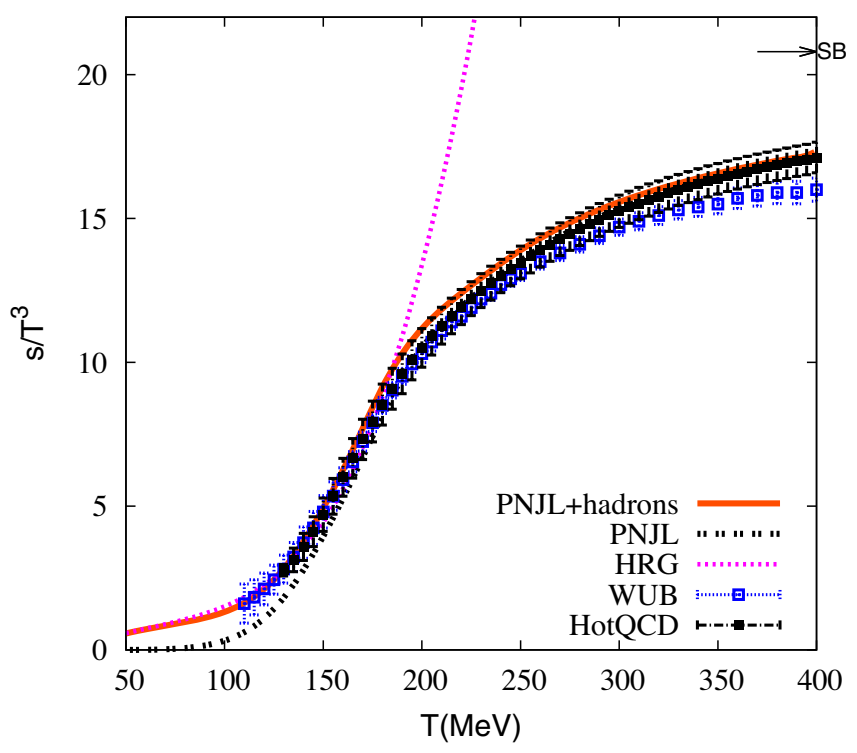

(b)

FIG. 2. Scaled pressure and entropy plotted as functions of temperature.

The scaled pressure and scaled entropy density are shown in Fig. 2(a) and Fig. 2(b) respectively. These are compared to the continuum extrapolated lattice QCD (HotQCD [2], and Wuppertal-Budapest [3]) data. Both quantities in the MPNJL model agree with the usual PNJL model and lattice QCD data for higher temperatures. At lower temperatures, the MPNJL model remarkably reproduces the lattice QCD data, where the PNJL model fails. Obviously a similar result was obtained with the hybrid PNJL model [15]. But unlike the hybrid model, where the switching function had to be tuned, here we have no extra parameters, apart from those already present in the PNJL model. As discussed previously, the use of temperature and medium dependent meson masses makes this picture an interactive one. The thermodynamic observables as well as fluctuations of conserved charges, thereby, have implicit dependencies on the PNJL fields as well.

Given that we have considered only a few mesons corresponding to the flavor $\mathrm{SU}(3)$ octet and the lowest lying nucleons, the agreement of the bulk thermodynamics in the MPNJL model and lattice QCD data is surprising. However as shown in the figures, the scaled pressure and scaled entropy density obtained in the ideal hadron resonance gas model have an excellent overlap with the MPNJL and lattice QCD data in the low temperature region. It therefore seems sufficient to include the limited number of hadrons for the present study.

With the MPNJL model, we now obtain the specific heat at fixed volume, which includes the second derivative of the thermodynamic potential with respect to temperature. The variation of the scaled specific heat with temperature is shown in Fig. 3(a). For higher temperatures, the lattice results are distinctly different. In fact the difference can be seen to be gradually increasing as we move from the scaled pressure to scaled entropy density to finally the scaled specific heat. As already mentioned in [16], we chose the parameters in the Polyakov loop potential to agree with the HotQCD data. In the lower temperature ranges, it is difficult to conclude if the results of the MPNJL model may be preferred over the PNJL model results when compared to the lattice QCD data.

To bring out the difference between the two models, we therefore consider the squared speed of sound, which turns out to be the ratio of the entropy to the specific heat at fixed volume. This is shown in Fig. 3(b). Here we see a wide difference between the PNJL and MPNJL model results for the lower temperatures. The MPNJL model results indeed agrees well with both the hadron resonance gas model, as well as the lattice QCD data.

With these results we demonstrated the importance of introducing beyond mean field contributions to the PNJL model, though with quite a few assumptions. As we see that here we did not need any extra switch between the hadron and PNJL contributions. The switch between the degrees of freedom are affected by the varying masses with temperature. For lower temperatures the constituent quark masses are quite high and the meson masses comparatively low, giving rise to meson domination. This is in addition to the suppression of quark excitation by the Polyakov loop. The condition is reversed as one approaches higher temperatures, and the system becomes quark dominated.

A proper determination of the state of strongly interacting matter at finite temperatures and chemical potentials requires knowledge of the fluctuations of conserved charges [50-53]. They also act as indicators of phase transition or crossover through which the system passes [45,54-63]. At a 


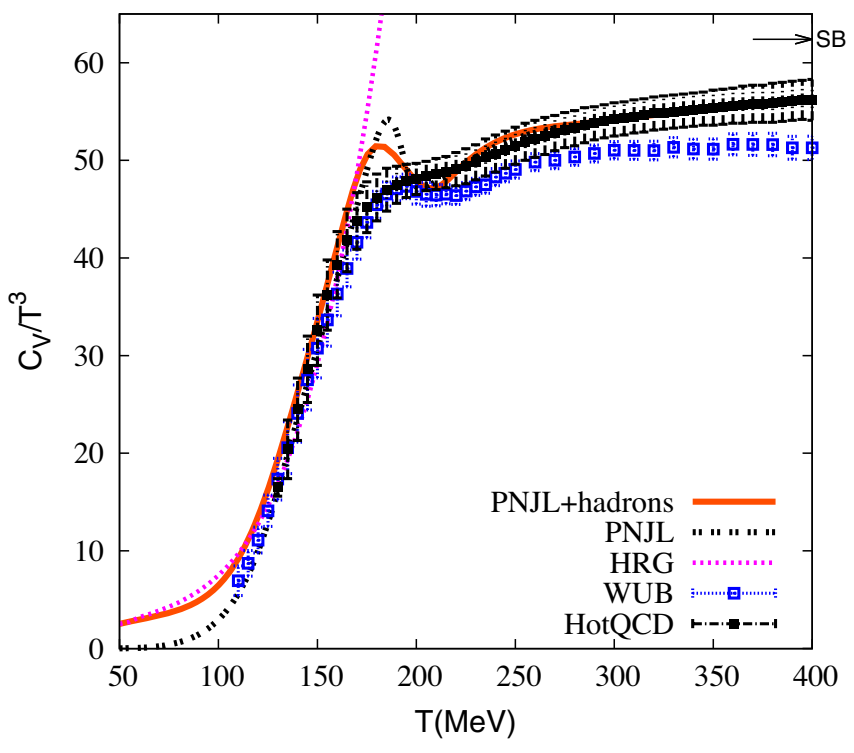

(a)

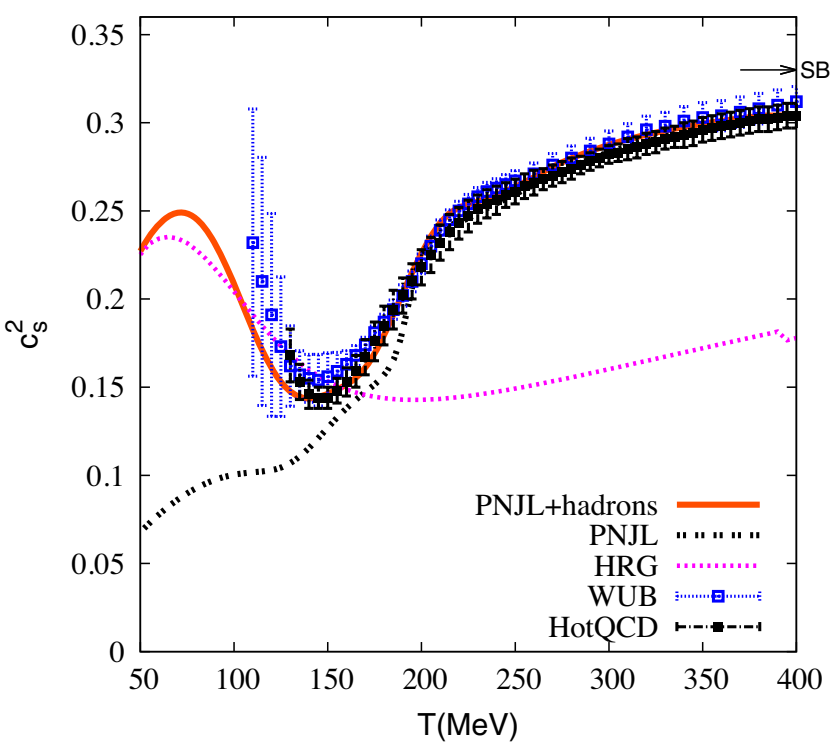

(b)

FIG. 3. Specific heat and speed of sound as functions of temperature.

given temperature and arbitrary chemical potentials, the pressure of the system may be expanded as a Taylor series around corresponding chemical potentials, where the coefficients are directly related by the fluctuation-dissipation theorem [64] to the fluctuations at various orders. The $n$th order Taylor expansion coefficient $c_{n}^{X}(T)$ of scaled pressure can be written in terms of fluctuations $\chi_{n}^{X}(T)$ of conserved charges (baryon number $B$, electric charge $Q$ and strangeness $S$ ) as,

$$
c_{n}^{X}(T)=\frac{1}{n !} \frac{\partial^{n}\left(P / T^{4}\right)}{\partial\left(\mu_{X} / T\right)^{n}}=\frac{T^{n-4}}{n !} \chi_{n}^{X}(T)
$$

where the expansion is carried out around $\mu_{B}=\mu_{Q}=\mu_{S}=0$. In Fig. 4 we present our results for the second order fluctuations of the conserved charges along with a comparison of the continuum data from lattice QCD [65-67]. In the model, these fluctuations are obtained by a suitable Taylor series fitting as discussed in detail in [21].

The baryon number fluctuation $c_{2}^{B}$ obtained in the PNJL and the MPNJL model are very close to each other in the whole range of temperatures studied. The only hadrons that contribute additionally in the MPNJL model are the nucleons with a mass $\sim 1 \mathrm{GeV}$, which is much heavier than the corresponding constituent mass of the quarks. So the difference between the two model results is insignificant. There is a possible concern for overcounting the baryons in the MPNJL model—as the constituent quarks and as the nucleons. Obviously this would be of concern as more and more baryons are included. But, in the present case, the constituent quarks overwhelm the system due to their lower masses as well as larger degrees of freedom. The issue of overcounting might also be significant while exploring the finite chemical potential scenario. The back reaction of hadrons desolving to quarks and antiquarks would be an important factor to deal with such situations. In view of these discussions, it is surprising to find a reasonable agreement of the results from the PNJL model with the lattice QCD data even for temperatures below $150 \mathrm{MeV}$. It seems that the partonic fluctuations manifest themselves strongly in the baryon susceptibilities.

This is not the case for the electric charge fluctuations $c_{2}^{Q}$. The PNJL and MPNJL model results differ significantly for temperatures close to $200 \mathrm{MeV}$. The MPNJL model has a very good agreement with the lattice QCD results. The dominant hadrons in this sector are the pions. For very low temperatures, the pion mass is almost half the mass of the constituent quarks. With increase in temperature, the pion mass increases and the quark mass decreases such that the combination nicely reproduces the lattice QCD data.

The strangeness fluctuation $c_{2}^{S}$ in the PNJL and MPNJL models differ for low temperatures by a smaller amount when compared to the charge fluctuations. For the lowest temperatures in the lattice QCD data, the MPNJL model seems to agree. But thereafter the two models merge and they deviate from the lattice QCD data. In the hadronic sector the dominant contributors are the $K$ and $\eta$. Their masses, though almost half of the constituent masses $M_{u}+M_{s}$, are still quite large. Moreover, the $K$ mass is almost constant over the whole temperature range. On the other hand, the decrease in the constituent mass of the strange quark with temperature is not fast enough. So their contributions to strangeness fluctuations above temperatures of $150 \mathrm{MeV}$ are not enough to agree with the lattice QCD data. Incorporation of higher order fermionic 

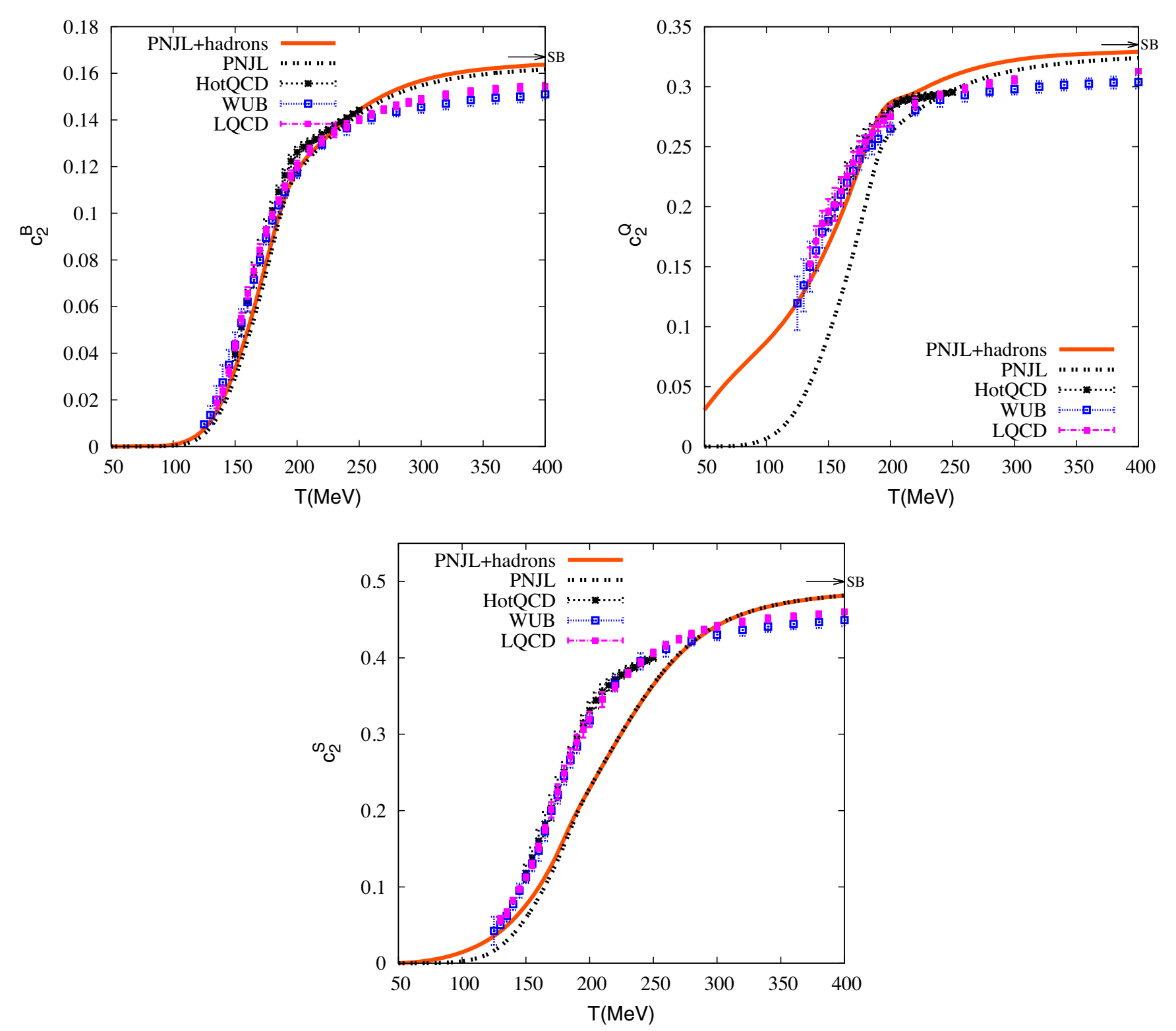

FIG. 4. Conserved charge fluctuations as functions of temperature.

interactions like 8-quark ones [16], along with massive strange hadrons (e.g., $\Lambda$ ) do not resolve the issue in the intermediate temperature range. These hadrons which are supposed to get excited in the intermediate temperature range under concern, do not quite contribute to the observables. As a matter of fact, the only way which remains to remove this artifact, is to reparametrize the NJL sector for strangeness. The current mass considered for strange quark as in Table I, is quite large compared to the lattice QCD observations. We plan to take care of these issues in our future projects.

\section{CONCLUSION}

Numerous attempts are being made to predict the correct EoS for strongly interacting systems. Lattice QCD is the most robust ab initio technique. However effective models, that are much easier to handle and suitable enough to extract interesting physical outcomes, are regularly employed. The reliability of such models in quantitative estimates have often come under review. In this regard, we are investigating the various possible improvements for the PNJL model so that it can serve as an effective tool for quantitative analysis of strong interactions in chemical equilibrium.

In an earlier work [16], the Polyakov loop potential was reparametrized to bring various thermodynamic quantities in reasonable agreement with the lattice QCD data. Among the issues pointed out in that work was the insufficiency of the PNJL model to reproduce the correct results for temperatures close to and below the crossover transition. The relevance of the hadronic degrees of freedom was realized and a hybrid model was built [15] with the HRG 
model and PNJL model coupled via a switching function. The method worked well, but a more natural framework was sought. The existing literature on the beyond-mean field calculations in the NJL and PNJL models led us to propose the present modified PNJL model, where the hadronic contributions are additively included in pressure. There is no switching function, but the hadrons are given medium modified masses. The relative variation of the hadron and constituent quark masses with temperature effectively selects the dominant degrees of freedom, thereby making this approach an interactive picture between the two phases of QCD matter. The best utility of this scheme over the scheme using switching function is for finite chemical potentials. The parameters in the switching function being additional parameters had to be fixed at various temperatures and chemical potentials. Here on the other hand there are no extra parameters and the effect of temperature and chemical potentials are taken care of through the respective hadronic distribution functions. In this first case study, we implement a simple approach. Instead of using the full MPNJL potential, which includes contributions from hadron sector, we minimize the thermodynamic potential given in Eq. (1). Our idea was to find whether, the hadronic masses can themselves act as inmedium switching functions. In the next step we plan to minimize the thermodynamic function including hadrons, more equivalent to a beyond mean field theory, which will be addressed in a future work.

The scheme is found to satisfactorily reproduce the lattice QCD results for a range of observables including the pressure, entropy, specific heat, speed of sound, and the baryon number and electric charge fluctuations. The results from the model however deviated significantly from the lattice data for strangeness fluctuations. To address this shortfall it is necessary to revisit the strangeness sector of the PNJL model, which we hope to address in the future.

\section{ACKNOWLEDGMENTS}

The authors would like to thank DST, DAE, Board of Research in Nuclear Sciences (BRNS), University Grants Commission (UGC). A. B. thanks Alexander von Humboldt $(\mathrm{AvH})$ foundation and Federal Ministry of Education and Research (Germany) for support through Research Group Linkage programme. K. S. acknowledges DST-SERB for financial support under NPDF file no. PDF/ 2017/002399. S. M. thanks CSIR for financial support. K. S. and S. U. would like to thank Souvik Priyam Adhya for useful discussions.
[1] A. Bazavov et al. (HotQCD Collaboration), Phys. Rev. D 85, 054503 (2012).

[2] A. Bazavov et al. (HotQCD Collaboration), Phys. Rev. D 90, 094503 (2014).

[3] S. Borsanyi, Z. Fodor, C. Hoelbling, S. D. Katz, S. Krieg, and K. K. Szabo, Phys. Lett. B 730, 99 (2014).

[4] Y. Nambu and G. Jona-Lasinio, Phys. Rev. 122, 345 (1961); 124, 246 (1961).

[5] T. Kunihiro and T. Hatsuda, Phys. Lett. B 206, 385 (1988).

[6] U. Vogl and W. Weise, Prog. Part. Nucl. Phys. 27, 195 (1991).

[7] S. P. Klevansky, Rev. Mod. Phys. 64, 649 (1992).

[8] T. Hatsuda and T. Kunihiro, Phys. Rep. 247, 221 (1994).

[9] M. Buballa, Phys. Rep. 407, 205 (2005).

[10] K. Fukushima, Phys. Lett. B 591, 277 (2004).

[11] R. D. Pisarski, Phys. Rev. D 62, 111501 (2000); A. Dumitru and R. D. Pisarski, Phys. Lett. B 504, 282 (2001); Phys. Rev. D 66, 096003 (2002).

[12] C. Ratti, M. A. Thaler, and W. Weise, Phys. Rev. D 73, 014019 (2006).

[13] K. Fukushima, Phys. Rev. D 77, 114028 (2008).

[14] H. Hansen, W. M. Alberico, A. Beraudo, A. Molinari, M. Nardi, and C. Ratti, Phys. Rev. D 75, 065004 (2007).

[15] A. Bhattacharyya, S. K. Ghosh, S. Maity, S. Raha, R. Ray, K. Saha, S. Samanta, and S. Upadhaya, Phys. Rev. C 99, 045207 (2019).
[16] A. Bhattacharyya, S. K. Ghosh, S. Maity, S. Raha, R. Ray, K. Saha, and S. Upadhaya, Phys. Rev. D 95, 054005 (2017).

[17] S. Borsanyi, Z. Fodor, C. Hoelbling, S. D. Katz, S. Krieg, C. Ratti, and K. K. Szabo, J. High Energy Phys. 09 (2010) 073.

[18] E. Megias, E. R. Arriola, and L. L. Salcedo, Phys. Rev. Lett. 109, 151601 (2012).

[19] A. Miyahara, Y. Torigoe, H. Kouno, and M. Yahiro, Phys. Rev. D 94, 016003 (2016).

[20] P. N. Meisinger and M. C. Ogilvie, Phys. Lett. B 379, 163 (1996); Nucl. Phys. B, Proc. Suppl. 47, 519 (1996).

[21] S. K. Ghosh, T. K. Mukherjee, M. G. Mustafa, and R. Ray, Phys. Rev. D 73, 114007 (2006).

[22] S. Mukherjee, M. G. Mustafa, and R. Ray, Phys. Rev. D 75, 094015 (2007).

[23] S. K. Ghosh, T. K. Mukherjee, M. G. Mustafa, and R. Ray, Phys. Rev. D 77, 094024 (2008).

[24] M. Ciminale, R. Gatto, N. D. Ippolito, G. Nardulli, and M. Ruggieri, Phys. Rev. D 77, 054023 (2008).

[25] A. Bhattacharyya, P. Deb, S. K. Ghosh, and R. Ray, Phys. Rev. D 82, 014021 (2010).

[26] G. Y. Shao, Z. D. Tang, M. Di Toro, M. Colonna, X. Y. Gao, and N. Gao, Phys. Rev. D 94, 014008 (2008).

[27] G. Y. Shao, Z. D. Tang, M. Di Toro, M. Colonna, X. Y. Gao, N. Gao, and Y. L. Zhao, Phys. Rev. D 92, 114027 (2015).

[28] C. A. Islam, S. Majumder, N. Haque, and M. G. Mustafa, J. High Energy Phys. 02 (2015) 011. 
[29] S. Ghosh, T. C. Peixoto, V. Roy, F. E. Serna, and G. Krein, Phys. Rev. D 93, 045205 (2016).

[30] J. Moreira, B. Hiller, A. A. Osipov, and A. H. Blin, Int. J. Mod. Phys. A 27, 1250060 (2012).

[31] C. Ratti, S. Robner, and W. Weise, Phys. Lett. B 649, 57 (2007).

[32] K. Fukushima, Phys. Rev. D 77, 114028 (2008); 78, 039902 (E) (2008).

[33] G. A. Contrera, A. G. Grunfeld, and D. B. Blaschke, Phys. Part. Nucl. Lett. 11, 342 (2014).

[34] X. Xin, S. Qin, and Y. Liu, Phys. Rev. D 89, 094012 (2014).

[35] J. Hufner, S. P. Klevansky, and P. Zhuang, Ann. Phys. (N.Y.) 234, 225 (1994).

[36] P. Zhuang, J. Hufner, and S. P. Klevansky, Nucl. Phys. A576, 525 (1994).

[37] A. Wergieluk, D. Blaschke, Y. L. Kalinovsky, and A. V. Friesen, Phys. Part. Nucl. Lett. 10, 660 (2013).

[38] S. Rossner, T. Hell, C. Ratti, and W. Weise, Nucl. Phys. A814, 118 (2008).

[39] A. E. Radzhabov, D. Blaschke, M. Buballa, and M. K. Volkov, Phys. Rev. D 83, 116004 (2011).

[40] K. Yamazaki and T. Matsui, Nucl. Phys. A913, 19 (2013); 922, 237 (2014).

[41] D. Blaschke, M. Buballa, A. Dubinin, G. Ropke, and D. Zablocki, Ann. Phys. (Amsterdam) 348, 228 (2014).

[42] J. M. Torres-Rincon and J. Aichelin, Phys. Rev. C 96, 045205 (2017).

[43] A. L. Fetter and J. D. Walecka, Quantum Theory of Many Particle System (McGraw-Hill, New York, 1971).

[44] P. Deb, A. Bhattacharyya, S. Datta, and S. K. Ghosh, Phys. Rev. C 79, 055208 (2009).

[45] A. Bhattacharyya, P. Deb, S. K. Ghosh, R. Ray, and S. Sur, Phys. Rev. D 87, 054009 (2013).

[46] H. Leutwyler and A. V. Smilga, Nucl. Phys. B342, 302 (1990).

[47] T. Sasaki, N. Yasutake, M. Kohno, H. Kouno, and M. Yahiro, arXiv:1307.0681v2.
[48] P. Costa, M. C. Ruivo, C. A. de Sousa, H. Hansen, and W. M. Alberico, Phys. Rev. D 79, 116003 (2009).

[49] P. Costa, M. C. Ruivo, and C. A. de Sousa, Phys. Rev. D 77, 096009 (2008).

[50] S. Ejiri, F. Karsch, and K. Redlich, Phys. Lett. B 633, 275 (2006).

[51] A. Bhattacharyya, P. Deb, A. Lahiri, and R. Ray, Phys. Rev. D 82, 114028 (2010).

[52] A. Bhattacharyya, P. Deb, A. Lahiri, and R. Ray, Phys. Rev. D 83, 014011 (2011).

[53] B. Abelev et al., Phys. Rev. Lett. 110, 152301 (2013).

[54] Y. Hatta and M. A. Stephanov, Phys. Rev. Lett. 91, 102003 (2003).

[55] A. Bhattacharyya, S. Das, S. K. Ghosh, S. Raha, R. Ray, K. Saha, and S. Upadhaya, arXiv:1212.6010v1.

[56] S. Jeon and V. Koch, Phys. Rev. Lett. 83, 5435 (1999).

[57] S. Jeon and V. Koch, Phys. Rev. Lett. 85, 2076 (2000).

[58] M. Asakawa, U. Heinz, and B. Muller, Phys. Rev. Lett. 85, 2072 (2000).

[59] A. Bhattacharyya, S. Das, S. K. Ghosh, R. Ray, and S. Samanta, Phys. Rev. C 90, 034909 (2014).

[60] A. Bhattacharyya, R. Ray, and S. Sur, Phys. Rev. D 91, 051501 (2015).

[61] A. Bhattacharyya, R. Ray, S. Samanta, and S. Sur, Phys. Rev. C 91, 041901 (2015).

[62] A. Bhattacharyya, S. K. Ghosh, R. Ray, K. Saha, and S. Upadhaya, Europhys. Lett. 116, 52001 (2016).

[63] M. A. Stephanov, K. Rajagopal, and E. V. Shuryak, Phys. Rev. Lett. 81, 4816 (1998).

[64] S. K. Ghosh, A. Lahiri, S. Majumder, M. G. Mustafa, S.Raha, and R. Ray, Phys. Rev. D 90, 054030 (2014).

[65] A. Bazavov et al., Phys. Rev. D 86, 034509 (2012).

[66] S. Borsanyi, Z. Fodor, S. D. Katz, S. Krieg, C. Ratti, and K. Szabo, J. High Energy Phys. 01 (2012) 138.

[67] R. Bellwied, S. Borsanyi, Z. Fodor, S. D. Katz, A. Pasztor, C. Ratti, and K. K. Szabo, Phys. Rev. D 92, 114505 (2015). 